\title{
LA-UR-03-0580
}

Approved for public release; distribution is unlimited.

\author{
Title: $\mid$ RADIOLYTIC EFFECTS OF PLUTONIUM \\ Author(s): Z.P. Zagorski ${ }^{\star}$, J. Dziewinski ${ }^{\star \star}$, and J. Conca ${ }^{\star \star \star}$ \\ *Institute of Nuclear Chemistry and Technology, Warsaw, \\ Poland \\ **Los Alamos National Laboratory, Los Alamos, NM, USA \\ ***Los Alamos National Laboratory, Carlsbad Operations, \\ NM, USA \\ Submitted to: \\ Plutonium Futures - The Science 2003 \\ A Topical Conference on Plutonium and Actinides
}

Los Alamos National Laboratory, an affirmative action/equal opportunity employer, is operated by the University of California for the U.S. Department of Energy under contract W-7405-ENG-36. By acceptance of this article, the publisher recognizes that the U.S. Government retains a nonexclusive, royalty-free license to publish or reproduce the published form of this contribution, or to allow others to do so, for U.S. Government purposes. Los Alamos National Laboratory requests that the publisher identify this article as work performed under the auspices of the U.S. Department of Energy. Los Alamos National Laboratory strongly supports academic freedom and a researcher's right to publish; as an institution, however, the Laboratory does not endorse the viewpoint of a publication or guarantee its technical correctness. 


\author{
RADIOLYTIC EFFECTS OF PLUTONIUM \\ Z.P. Zagorski*, J. Dziewinski**, and J. Conca*** \\ *Institute of Nuclear Chemistry and Technology, Warsaw, Poland \\ **Los Alamos National Laboratory, Los Alamos, New Mexico, USA, \\ ***Los Alamos National Laboratory, Carlsbad Operations, New Mexico, USA,
}

\title{
Introduction
}

Plutonium isotopes, most of them $\alpha$-emitters, cause radiolytic changes in the matrix, in which they are embedded. The internal irradiation of $\mathrm{Pu}^{0}$ metal or its alloys results in physical changes, largely as a result of the formation of helium bubbles, well-known to material scientists and weapons specialists. In all other media where plutonium occurs, usually as $\mathrm{Pu}^{\mathrm{n}+}$ in an ionic form, the results of irradiation are chemical in nature. Homogenous media containing $\mathrm{Pu}$, are often aqueous or non-aqueous solutions of plutonium compounds, mostly originating during processing of spent nuclear fuel or from Pu processing. Heterogenous matrices containing plutonium are more complex from the point of view of radiolysis; they usually contain a variety of combinations of common materials contaminated with radionuclides. This class of radioactive materials represents a challenge for the management of plutonium waste. One has to consider a range of time scales for radiolytic effects (and consequently a several orders of magnitude range of the cumulative dose) beginning with waste generation, through packaging, transportation, to the period of final storage. Final storage could be for thousands of years in deep geologic repositories. At every 'stage of that time scale, radiolysis proceeds continuously and cumulative effects can complicate operating procedures and final disposition. The results presented here have been obtained from experiments that have irradiated of model materials, which are typically the objects of contamination with plutonium. They were irradiated with linearly accelerated electrons up to very high dose rates, adjusted to simulate any contamination at any point on the time scale. 
The wide field of all possible interactions of plutonium radiation with very different chemical compounds has been divided into groups of problems ordered according to the urgency of occurring in practice. The most needed information was about the radiation yield of hydrogen from the fastest decaying plutonium isotopes. That information is influencing the strategy of preparation of waste for transportation. The problem involves investigating various materials, such as organic compounds, including polymers, concretes containing plutonium, and plutonium-contaminated organic debris. Another set of problems includes salt rock, in geologic salt repositories, which will eventually come into contact with plutonium after the containment corrodes over the course of thousands of years. Salt exposed to radiation can form electron traps in the crystalline lattice defects. The worst-case scenario involved in modeling of the repositories assumes that water will seep into the deposit with irradiated salt. The resulting dissolution of F-centers will free the trapped electrons and cause radiolysis of the brine, separate from radiolysis that will be occurring in the waste. The spectrum of products of this radiolysis is wide and complicated. Besides many chlorine-oxygen compounds formed, also hydrogen peroxide appears in multi-ionization spurs of comparatively high LET radiation. The seeping water may, but does not have to be, deoxygenated by iron and organic materials present in the waste. The presence or absence of oxygen has a tremendous effect on the outcome of radiolysis and both conditions need to be investigated.

A $10 \mathrm{MeV}$ electron beam (EB) has been applied as the source of radiation in these investigations. It simulates plutonium $\alpha$ radiation, by taking into account easy recalculable effects of different LET radiations. An integrated hardware system of EB irradiation combined with gas chromatography has been developed to handle specific difficulties occurring with the delivery of wide dose ranges, stretching over several orders of magnitude.

It is very important from waste management perspective to investigate $\mathrm{H}_{2}$ production by irradiation, which occurs in all chemical systems containing hydrogen. Hydrogen is energetically easiest 
to detach, whether bound inorganically or present in organic compounds, especially in polymers. Hydrogenated butadiene rubber has been chosen as a model polymer for elastomers often occurring in the waste as gaskets or tubings. As theoretically expected, a linear relationship of $\mathrm{H}_{2}$ production versus dose was observed beyond the initial dose of $20 \mathrm{kGy}$ (Fig.1). The reduced yield of hydrogen generation in the initial stage is caused by aromatic additives used routinely in the polymer industry. The phenomenon is welcomed from the point of view of waste management, because it means that the hydrogen generation in the initial stage of waste disposition, i.e. transportation, is less than in the later stages of radiolysis. Other polymers, both synthetic and natural (e.g. cellulose) are under investigation and very high doses have been administered.

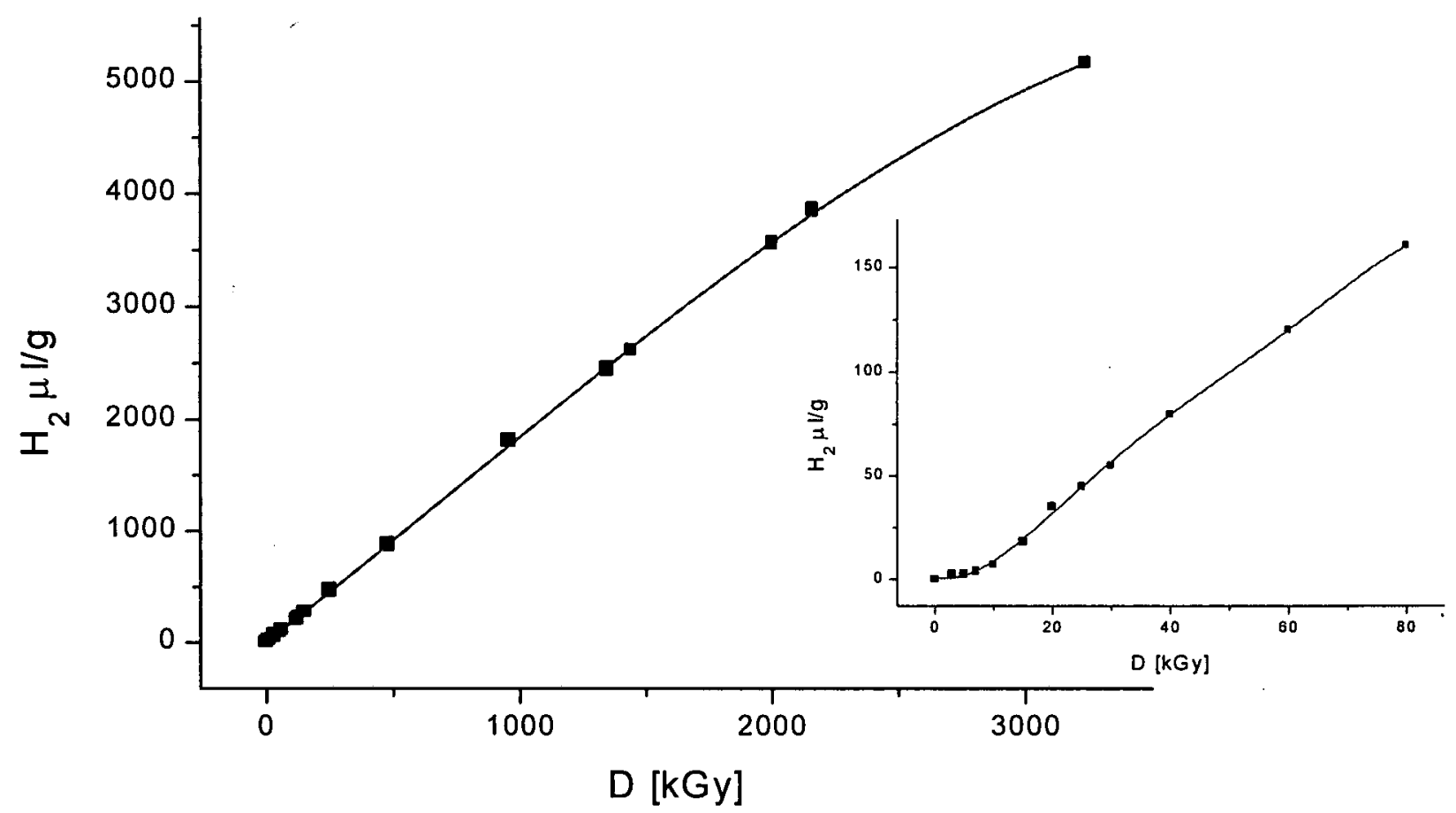

Fig. 1. Production of hydrogen ( $\mu \mathrm{L}$ normal) per $1 \mathrm{~g}$ of elastomer in the function of dose. Insert: Production of hydrogen at low doses, showing diminished yield due to the presence of stabilizing additives. 
Slight changes in the technique is applied to the determination of hydrogen yield from concrete containing plutonium and various organics, involved in processing of nuclear fuel and recovery of plutonium from weapons. Although no chemical chain reaction of hydrogen generation has been observed, that question is still open and will be eventually answered as research continuous. There is a possibility of a chemical chain reactions in the presence of some materials. Such materials should be avoided during plutonium processing. Different procedures are applied for experimental investigation of radiolysis of brines. The analyses of irradiated brines have shown the sensitivity of the radiolysis to the presence of organics (possible humic acids in seeping water) and to the presence of oxygen. Preliminary results were presented and discussed on two international conferences in 2002 [1,2].

\section{Discussion and Conclusions}

Investigating the chemical aspects of radiation of plutonium is important for waste management for several reasons. It singles out the risks related to the radiolytic products and indicates ways to mitigate and avoid those risks. It also lowers the expense of waste management, by lowering the maximum limits of safe packaging, transportation and storage of waste. The reported investigations, continued in 2003, fit well into the increasing role of radiation chemistry in radioactive waste management, as announced by Zimbrick [3]. Plutonium future is hard to imagine without considerations of radiolytic phenomena occurring when nuclides are in contact with, and embedded in, waste materials. It is part of the responsibility of our generations towards next generations to come.

Acknowledgment. This work is made and continued under subcontract LANL-INCT 45302-001-02-AA.

Keywords:

brines radiolysis, hydrogen generation, plutonium radiation, polymer radiolysis, radiolysis, spurs 


\section{References}

[1]. Zagórski, Z.P., 14 ${ }^{\text {th }}$ Radiochemical Conference, 14-19 April 2002, Marianske Lazne, Czech Republic, two oral presentations: a: Radiation chemistry in the management of radioactive waste (selected topics), b: Spectroscopic investigation of the formation of radiolysis products by $10 \mathrm{MeV}$ linear accelerator electron in salt solutions.

[2]. Zagórski, Z.P., Dziewinski, J., $5^{\text {th }}$ International Symposium on Ionizing Radiation and Polymers (IraP 2002), Sept. 21-26, 2002, Saint-Adele (Quebec) Canada, Paper: Radiation chemistry of polymeric components of radioactive waste.

[3]. Zimbrick, J.D., Radiation Research 158, 127-140 (2002). 